\title{
Effect of Foliar Zinc Application on Yield, Physiological Traits and Seed Vigor of Two Soybean Cultivars under Water Deficit
}

\author{
Somayeh KARAMI, Seyed Ali Mohammad MODARRES SANAVY*, \\ Sanam GHANEHPOOR, Hamed KESHAVARZ
}

Tarbiat Modares University Tehran, Faculty of Agriculture, Agronomy Department, Iran; modarresa@yahoo.com ("corresponding author)

\begin{abstract}
In order to study the effect of water deficit stress and zinc foliar application on yield, physiological traits and also on seed vigor and seedling emergence percentage in two soybean cultivars, an experiment was conducted as randomized complete block arrangement in split factorial design with three replications. The main factor was drought stress in three levels of optimal irrigation, withholding irrigation from vegetative growth stage and withholding irrigation from flowering stage; subordinate factors were the combination of foliar zinc application in three levels and two cultivars ('L17' and 'Clark 63'). Water deficit stress obviously decreased the yield, soluble protein and chlorophyll content in leaves. Proline and soluble sugars content were significantly increased in response to stress. Water deficit stress increased antioxidant enzymes activity. Also, water deficit stress decreased the germination rate, radicle and plumule dry weight. Foliar application with zinc sulfate increased the yield, germination rate and percentage, radicle and plumule weight. Zinc prevented the harmful effects of stress which caused decreasing of leaf protein, chlorophyll content and increasing proline and carbohydrate accumulation. In general, foliar application of zinc decreased the harmful effects of oxidative stress due to water deficit stress and improved growth conditions of plants.
\end{abstract}

Keywords: physiological traits, seed vigor, soybean, water deficit, yield

\section{Introduction}

On a global basis, drought, in conjunction with high temperature and radiation, poses the most fundamental environmental constraints to plant survival and crop productivity (Chaves et al., 2003). Changes in water balance and the amount of available water in soil can be crucial for crop yield (Fuhrer, 2003). Water deficit is also known to alter a variety of biochemical and physiological processes, ranging from photosynthesis to protein synthesis and solute accumulation (Hu and Schmidhalter, 1998). Increasing evidence has indicated that much of the plants injuries due to various stresses are associated with oxidative damage through direct or indirect formation of active oxygen species (AOS). These AOS attack lipids, proteins, chloroplasts and nucleic acids, causing lipid peroxidation, protein denaturation and DNA mutation. To prevent such damages, plants have an antioxidative and protective enzymatic system including superoxide dismutases (SODs), catalases (CATs) and peroxidases, to scavenge and protect cells against detrimental injuries of free radicals (Bowler $e t$ al., 1992). Osmotically active solutes and soluble protein do not possess direct osmotic adjustment roles as compatible solutes, but hold protection functions to biological macromolecules against oxidative damage (Taiz and Zeiger, 1991).

Inadequate and unbalanced supply of mineral nutrients (micro and macronutrients) and impaired soil fertility are particular problems, causing decreases in food production, especially in the developing countries. Micronutrients are essential for normal physiological activity of plants (Bai, 1992), as these have the same importance as macronutrients (Razmjoo and Henderlong, 1997). Among micronutrients, zinc $(\mathrm{Zn})$ is essential for growth, development and crops productivity. $\mathrm{Zn}$ role is either as metal component of various enzymes or as a functional, structural or regulatory cofactor and is thus linked with photosynthesis and carbohydrate metabolism (Farahat et al., 2007). Zn deficiency is a widespread micronutrient disorder in different agroclimatic regions of the world, particularly within arid and semi-arid areas (White and Zasoski, 1999). The major reason for the widespread occurrence of $\mathrm{Zn}$ deficiency in soils is low availability of $\mathrm{Zn}$ to plant roots, rather than low $\mathrm{Zn}$ content in soils. High $\mathrm{pH}$ and high levels of $\mathrm{CaCO}_{3}$, as well as low levels of organic matter are predominantly responsible for the low availability of Zn to plant roots (Marschner, 1993).

Foliar application of microelements is more beneficial than soil application (Zayed et al., 2011). Undoubtedly, soybean yield and its oil would have higher quality by microelements foliar spraying. It was documented that $\mathrm{Zn}$ foliar application is a simple way for making quick correction of plant nutritional status, as reported for wheat (Erenoglu et al. 2002). Accumulating evidence suggests that mineral-nutrient status of plants plays a critical role in increasing plant ability to resist and adapt to adverse stress conditions (Marschner, 1995; Khan et al., 2003; 
182

2004). Because an adequate supply of mineral nutrients is indispensable for the maintenance of photosynthetic electron transport and carbon metabolism, impairment of the mineral nutritional status of plants under marginal environmental conditions can exacerbate photooxidative damage and limit plant performance (Marschner, 1995).

Zinc nutritional status of plants may affect their drought sensitivity (Cakmak, 2000). Applying Zn increased chickpea grain yield when the plants were well-watered, but not under water stress, except for $\mathrm{Zn}$-efficient and drought resistance genotype 'ICC-4955' (Khan et al., 2003). Foliar Zn and Mn application under drought stress can positively influence yield production and seed quality of safflower genotypes during different growth stages (Movaheddy-Dehnavy et al., 2009). Yilmaz et al. (1998) reported that yield of wheat plants grown from high seed-Zn content was $116 \%$ higher than the yield of plants grown from the low seed-Zn content under rain fed and $\mathrm{Zn}$-deficient soil. In chickpea, $\mathrm{Zn}$ deficiency reduced water use due to reduced plant growth and lower stomatal conductance and that water stress developed gradually as a consequence (Khan et al., 2004).

Despite this effect, Zn-deficient plants use water less efficiently and are less able to respond to increasing soil water deficits by osmotic adjustment than plants that are supplied with adequate levels of $\mathrm{Zn}$. It has been reported that positive effects of $\mathrm{Zn}$ application on photosynthesis and growth of maize were mostly found under sufficient water supply (Wang et al., 2009). Wang and Jin (2007) demonstrated that the higher AOS level in early maize leaves due to water stress was not influenced (alleviated or lowered partially) by $\mathrm{Zn}$ application. However, these researchers recommended that $\mathrm{Zn}$ fertilizer should be applied to maize plants irrigated or supplied with adequate water, or else $\mathrm{Zn}$ deficiency would reduce the water use for plant biomass production.

The current experiment was undertaken to examine the interactive effects between water stress and $\mathrm{Zn}$ nutrition on soybean plants (1) to reveal whether $\mathrm{Zn}$ foliar application would diminish AOS damages to soybean caused by drought stress; (2) to elucidate the effect of applied $\mathrm{Zn}$ on soybean and plants' adaptation to respond to water deficit conditions during different developmental stages, by determining some physiological indicators; (3) to study how grain yield and seed quality modify in regard with $\mathrm{Zn}$ nutritional status, under well and limited irrigation regimes.

\section{Materials and Methods}

\section{Site description, trial design and experimentation}

A three-factor experiment was conducted at the Agricultural Research Farm of Tarbiat Modarres University located in Karaj (35 $41^{\prime} \mathrm{N}$ latitude, $51^{\circ} 19^{\prime} \mathrm{E}$ longitude and altitudes of $1215 \mathrm{~m}$ ), Iran. Soil samples were collected at the depth of 30 and $60 \mathrm{~cm}$ and some physico-chemical properties were investigated. The soil was clay-sandy loam, alkaline (7.73), EC of $1.12 \mathrm{dS} \mathrm{m}$, low in organic matter (1.77\%), 0.01\% total nitrogen, $80 \mathrm{mg} \mathrm{P} \mathrm{kg}^{-1}, 884$ $\mathrm{mg} \mathrm{Kkg}$, and Zn-DTPA of $0.45 \mathrm{mg} \mathrm{kg}^{-1}$.

A factorial experiment $(3 * 3 * 2)$ was conducted in randomized complete block (RCB) design with split-plot factorial using three replications. The treatments comprised three irrigation levels $\left[\mathrm{I}_{1}=\right.$ well watering or control treatment, $\mathrm{I}_{2}$ $=$ stressed by skipping the irrigation at vegetative stage until $10 \%$ flowering $\left(V_{5}-R_{1}\right)$, and $I_{3}=$ irrigation disruption during flowering and $10 \%$ poding growth stages $\left.\left(\mathrm{R}_{1}-\mathrm{R}_{3}\right)\right]$, which were applied to main plots; the factorial combination of cultivars [' $\mathrm{L} 177^{\prime}\left(\mathrm{V}_{1}\right)$ and 'Clark63' $\left(\mathrm{V}_{2}\right)$ ] and foliar application [no application $\left(\mathrm{F}_{0}\right)$, foliar application of distilled water $\left(\mathrm{F}_{1}\right)$ and of zinc sulfate $\left.\left(\mathrm{F}_{2}\right)\right]$ were allocated to subplots.

Both of the tested cultivars were indeterminate. Seedbed was prepared by conventional tillage practices. The size of each subplot was $9.2 \mathrm{~m}^{2}$, consisted of 8 rows of $3 \mathrm{~m}$ length, spaced at $40 \mathrm{~cm}$ apart, while plant to plant distance was of $8 \mathrm{~cm}$. To avoid the effect of lateral movement of irrigation water, the main plots in each replication were separated by borders of $2 \mathrm{~m}$ in width from all sides. Seeds of soybean cultivars were inoculated with Rizobium japonicum strain immediately before seeding. At the same time, all experimental units were fertilized with $30 \mathrm{~kg} \mathrm{~N} \mathrm{~kg}^{-1}$ as urea. All the routine agronomic practices were done uniformly for the entire plots according to performed local practices.

In full irrigation treatment $\left(\mathrm{I}_{1}\right)$, irrigation was conducted as to equal the crop water requirement at the experimental site on the basis of $40 \%$ available water depletion that coincided with every three days supplementation; drought stressed plots were irrigated after $60 \%$ soil field capacity depletion, at the two mentioned growth stages.

Soil water content was determined by time domain reflectometry (TDR) device, so that water deficit was imposed uniformly in different stages. A hose with a counter on it and calibrated siphons were used to deliver the required amount of water. Before drought stress treatments imposition, foliar spray of zinc ( $\mathrm{as} \mathrm{ZnSO}_{4.7} .7 \mathrm{H}_{2} \mathrm{O}$ ) was twice applied on soybean cultivars foliage in two weeks intervals at the rate of $5 \mathrm{ppm}$. The first one was done at $4^{\text {th }}$ leaf stage.

\section{Extraction and assay of enzymes}

The youngest fully expanded leaf samples of both cultivars were frozen in liquid nitrogen immediately after harvesting and stored at $-80^{\circ} \mathrm{C}$ until enzyme analysis. Tested plant material (0.2 g of frozen samples) was grounded in $25 \mathrm{mM}$ sodium phosphate ( $\mathrm{pH}$ 6.8) for CAT, $0.02 \mathrm{M}$ potassium phosphate $(\mathrm{pH}$ 6.8) for POD and HEPES-KOH containing 0.01 mM EDTA (pH 7.8) buffers with a pastel in an ice-cold mortar. The homogenates were centrifuged at $2-4^{\circ} \mathrm{C}$ for $15 \mathrm{~min}$ at $12,000 \times \mathrm{g}$. Supernatant was used to measure the enzyme activity and protein content assays. Total soluble protein contents of the enzyme extracts were determined according to Bradford (1976) using bovine serum albumin as the standard.

CAT activity was assayed by the method of Cakmak and Engels (1999). Samples of $0.5 \mathrm{~g}$ of frozen plant tissues was homogenized in a mortar and pestle with $3 \mathrm{~mL}$ ice-cold extraction buffer ( $25 \mathrm{mM}$ sodium phosphate, $\mathrm{pH} 7.8$ ). The homogenate was centrifuged at $18,000 \mathrm{rpm}$ for $30 \mathrm{~min}$ at $4{ }^{\circ} \mathrm{C}$, and then the supernatant was used for enzyme assay. The reaction mixture contained $100 \mathrm{lL}$ crude enzyme extract, $500 \mathrm{lL}$ $10 \mathrm{mM} \mathrm{H} 2 \mathrm{O} 2$ and 1,400 IL $25 \mathrm{mM}$ sodium phosphate buffer. The decrease in the absorbance at $240 \mathrm{~nm}$ was recorded for 1 min by spectrophotometer. CAT activity of the extract was expressed as CAT units min-1 mg-1 protein.

SOD activity was assayed according to the method of Giannopolitis and Ries (1977). The reaction mixture contained $1,500 \mu \mathrm{l}$ of $50 \mathrm{mM}$ Hepes-KOH, $0.1 \mathrm{mM}$ EDTA, $300 \mu \mathrm{l}$ of $\mathrm{Na}_{2} \mathrm{CO}_{3}$ (pH 10.2), $300 \mu \mathrm{l}$ of $12 \mathrm{mM}$ L-methionin, $300 \mu \mathrm{l}$ of $75 \mu \mathrm{M}$ nitroblue tetrazolium, $300 \mu \mathrm{l}$ of $1 \mu \mathrm{M}$ riboflavin and $300 \mu \mathrm{l}$ of enzyme extract. SOD activity was assayed by measuring the ability of the enzyme extract to inhibit the photochemical 
reduction of NBT. Glass test tubes containing the mixture were illuminated with a fluorescent lamp $(120 \mathrm{~W})$; identical tubes that were not illuminated served as blanks. After illumination for $15 \mathrm{~min}$, the absorbance was measured at $560 \mathrm{~nm}$. One unit of SOD was defined as the amount of enzyme activity that was able to inhibit by $50 \%$ the photoreduction of NBT to blue formazan. The SOD activity of the extract was expressed as SOD units min-1 mg-1 protein.

POX activity was assayed by the oxidation of guaiacol in the presence of $\mathrm{H}_{2} \mathrm{O}_{2}$. The increase in absorbance was recorded at $470 \mathrm{~nm}$ (Ghanati et al., 2002). The reaction mixture contained $100 \mathrm{lL}$ crude enzyme, $500 \mathrm{IL} \mathrm{H} 2 \mathrm{O} 25 \mathrm{mM}, 500 \mathrm{lL}$ guaiacol 28 $\mathrm{mM}$ and 1,900 $\mathrm{IL}$ potassium phosphate buffer $60 \mathrm{mM}$ (pH 6.1). POX activity of the extract was expressed as POX units min-1 mg-1 protein.

\section{Proline content}

Leaves free proline content was extracted from $0.2 \mathrm{~g}$ of leaf samples in 3\% (w/v) aqueous sulphosalysylic acid and estimated by using ninhydrin reagent according to Bates et al. (1973). The absorbance of fraction with toluene aspired from liquid phase was read at $520 \mathrm{~nm}$. Proline concentration was determined using calibration curve and expressed as $\mathrm{mg}$ proline $\mathrm{g}^{-1} \mathrm{FW}$.

\section{Leafsoluble carbohydrates}

Carbohydrate fractions were extracted in distilled water, clarified and determined as total soluble sugars $\left(\mu \mathrm{gg}^{-1} \mathrm{FW}\right)$, based on phenol-sulfuric acid assay (Dubios et al., 1965). A volume of $0.5 \mathrm{~mL}$ of $5 \%(\mathrm{v} / \mathrm{v})$ phenol solution and $2.5 \mathrm{~mL}$ of concentrated sulfuric acid $98 \%(\mathrm{v} / \mathrm{v})$ were added to $50 \mu \mathrm{L}$ aliquots. The mixture was shaken, heated in a boiling water-bath for $20 \mathrm{~min}$ and cooled to room temperature. The absorption was then determined by spectrophotometry at $490 \mathrm{~nm}$. Standard curve was expressed as 0 to $10 \mu \mathrm{g} \mathrm{ml}^{-1}$ concentrations of xylose and manose.

\section{Pigment analysis}

Chlorophyll $a$ (chl a) and $b$ (ch b) were measured by Arnon's method (1949). In short, fresh leaf tissue $(0.2 \mathrm{~g})$ was homogenized in $80 \%$ cold acetone $(\mathrm{w} / \mathrm{v})$, passed through filter papers and a final volume of $25 \mathrm{ml}$ was reached by adding acetone. The absorbance of aliquot was recorded at 663 and 665 . The photosynthetic pigment content ( $\mathrm{mg} \mathrm{g}^{-1} \mathrm{FW}$ ) was quantified using the equations of Arnon (1949).

\section{Chlorophyll fluorescence}

Chlorophyll fluorescence parameters were recorded in parallel to above mentioned traits, using a portable fluorometer (Bio Monitor, AB, Sweden). Leaves acclimated to dark for 30 min before measurements were taken. The time of measuring was $5 \mathrm{~s}$; irradiance was set at $3,000 \mu \mathrm{mol} \mathrm{m} \mathrm{m}^{-1}$. Initial $\left(\mathrm{F}_{0}\right)$, maximum $\left(\mathrm{F}_{\mathrm{m}}\right)$ and variable $\left(\mathrm{F}_{\mathrm{v}}=\mathrm{F}_{\mathrm{m}}-\mathrm{F}_{0}\right)$ fluorescence were recorded. Fv/Fm was used to indicate the potential maximal quantum yield of photosystem II (PS II).

\section{Grain yield}

At maturity, plants of $4.4 \mathrm{~m}^{2}$ in the middle part of each plot were harvested and grain yield per unit area was determined.

\section{Seed quality and germination parameters}

At mass maturity when the moisture content of cultivars reached $12 \%$, seed samples of plants from $4.4 \mathrm{~m}^{2}$ in each treatment were detached. After determining Zn content, seeds were used for germination tests. For standard seed germination, ten seeds per cultivar were surface sterilized, and then sown between double layered rolled and moist filter papers, in three replications, according to ISTA (2008). The towel papers with seeds were put into plastic dishes to inhibit moisture loss, and then kept in germinator, maintaining a photoperiod of 16:8 (day/night) and adjusted temperatures of $30^{\circ} \mathrm{C}$ and $20^{\circ} \mathrm{C}$ for 10 days. Germination was recorded in daily intervals from day of planting seeds in the medium till the time when germination was stopped.

Mean time to germination (MTG) was calculated as $\frac{\sum n D}{\sum n}$ (Roberts and Ellis, 1980) to assess the germination rate $\left(R^{=}=\frac{1}{M T G}\right)$, where ' $\mathrm{n}$ ' is germinated seed numbers on day $\mathrm{D}$, ' $\mathrm{D}$ ' is the number of days from the test beginning and ' $\sum \mathrm{n}$ ' is total number of germinated seeds; ' $\mathrm{R}^{-}$' is mean germination rate.

At the end of the germination test $\left(10^{\text {th }}\right.$ day), normal and abnormal seedlings were counted and germination percentage was calculated as $\frac{\text { No.ofseedsger min atedseeds }}{\text { No.ofseesdssown }} \times 100$

Among normal seedlings radicles and shoots of 10 randomly selected seedlings were cut from the cotyledons and then dried in an oven at $75 \pm 2{ }^{\circ} \mathrm{C}$ for 24 hours. The dried radicles and shoots were weighed to the nearest milligram and the mean radicle and shoot dry weight and consequently mean seedling dry weight were determined. Seedling vigor index (SVI) was calculated by multiplying germination percentage and seedling dry weight (Abdul-Baki and Anderson, 1973).

\section{Statistical analysis}

Analysis of variance of all data appropriate to the experimental design was carried out by SAS software and the differences among the means were compared using LSD test at 0.01 probability level.

\section{Results and Discussion}

Total soluble protein and activities of some enzymes scavenging $A O S$

The main effects of all the experimental factors and two-way interaction between them were significant for the measured biochemical indicators, except for foliar $\mathrm{Zn}$ application $\times$ cultivar on activity total leaf soluble protein and activity of CAT and POD (Table 1). There was a general decreasing trend in leaf protein content of both cultivars due to water deficit (Table 3). However, $\mathrm{Zn}$ application could compensate this reduction up to $15 \%$ (Table 2). The differential response of cultivars to induced drought stress was obvious. The relatively high sensitivity of protein metabolism in 'L17' lead to a marked reduction of protein content at both vegetative and reproductive stages, as compared with 'Clark63', while when the soil moisture was sufficient, this cultivar had higher leaf protein content (Table 3 ).

The alternation of protein synthesis or degradation is one of the fundamental metabolic processes that may influence water stress tolerance (Wang and Huang, 2004). Both quantitative and qualitative changes of proteins have been detected during stress periods (Ahire et al., 2005; 
Table. 1. Analysis of variance on yield, physiological traits and seed vigor of two soybean cultivars

\begin{tabular}{|c|c|c|c|c|c|c|c|c|c|}
\hline S.O.V & $\begin{array}{l}\text { Soluble } \\
\text { protein }\end{array}$ & $\begin{array}{c}\text { Superoxide } \\
\text { dismutase }\end{array}$ & Peroxidase & Catalase & $\begin{array}{c}\text { Chlorophyll } \\
a\end{array}$ & $\begin{array}{c}\text { Chlorophyll } \\
b\end{array}$ & $\begin{array}{c}\text { Total } \\
\text { chlorophyll }\end{array}$ & Proline & $\begin{array}{c}\text { Soluble } \\
\text { carbohydrates }\end{array}$ \\
\hline Replication & 0.010 & 0.018 & 0.0041 & 109.0 & 0.0007 & 0.0007 & 0.0022 & 0.34 & 1895 \\
\hline Stress & $3.561^{* *}$ & $37.674^{* *}$ & $51.2111^{* *}$ & $13345.8^{* *}$ & $1.9957^{* *}$ & $0.8132^{* *}$ & $5.3503^{* *}$ & $10530.74^{* *}$ & $161401616^{* *}$ \\
\hline Errora & 0.004 & 0.034 & 0.0021 & 29.1 & 0.0013 & 0.0003 & 0.0012 & 0.19 & 8388 \\
\hline Variety & $0.491^{\text {** }}$ & $23.285^{* *}$ & $37.4561^{* *}$ & $5126.2^{* *}$ & $0.2893^{* *}$ & $01738^{* *}$ & $0.9115^{* *}$ & 1.48 & $106583113^{* *}$ \\
\hline Foliar application & $0.238^{* *}$ & $3.811^{* *}$ & $5.4823^{* *}$ & $504.8^{*}$ & $0.1905^{* *}$ & $0.0334^{* *}$ & $0.3825^{* *}$ & $9029.48^{* *}$ & $5334236^{* *}$ \\
\hline Variety $\times$ stress & $0.176^{* *}$ & 0.025 & $0.7294^{* *}$ & $245.80^{*}$ & $0.0170^{* *}$ & $0.0028^{*}$ & $0.0143^{* *}$ & 1.11 & $612343^{* *}$ \\
\hline Foliar application $\times$ stress & 0.006 & $0.772^{* *}$ & $4.6678^{* *}$ & $419.21^{* *}$ & $0.0254^{* *}$ & $0.0047^{* *}$ & $0.0472^{* *}$ & $2175.32^{* *}$ & $10827112^{* *}$ \\
\hline Variety $\times$ foliar application & 0.000 & $0.878^{* *}$ & 0.0049 & 1.44 & $0.0027^{*}$ & 0.0008 & $0.0062^{*}$ & $10.05^{*}$ & $203528^{*}$ \\
\hline Variety $\times$ stress $\times$ foliar application & 0.003 & 0.013 & 0.0022 & 6.8 & 0.0016 & 0.0000 & 0.0015 & 2.99 & 72772 \\
\hline Errorb & 0.004 & 0.089 & 0.0054 & 68.2 & 0.0008 & 0.00005 & 0.0016 & 2.04 & 38438 \\
\hline $\mathrm{CV}(\%)$ & 2.85 & 4.85 & 1.46 & 5.09 & 2.66 & 4.71 & 2.620 & 4.58 & 5.01 \\
\hline S.O.V & $(\mathrm{fv} / \mathrm{fm})$ & $\begin{array}{l}\text { Germinatio } \\
\text { n percentage }\end{array}$ & $\begin{array}{c}\text { Rate of } \\
\text { Germination }\end{array}$ & $\begin{array}{c}\text { Radical dry } \\
\text { weight }\end{array}$ & $\begin{array}{l}\text { Shootdry } \\
\text { weight }\end{array}$ & Seedlingdry & $\begin{array}{l}\text { Seedlingvigour } \\
\text { Index }\end{array}$ & Grain yield & \\
\hline Replication & 0.00007 & 0.074 & 0.21 & 0.00000241 & 0.0000014 & 0.000000 & 0.109 & 882 & \\
\hline Stress & $0.07784^{* *}$ & $1187.85^{* *}$ & $146.09^{* *}$ & $0.00001820^{* *}$ & $0.0000440^{* *}$ & $0.000120^{* *}$ & $7.591^{* *}$ & $3658406^{* *}$ & \\
\hline Errora & 0.00013 & 15.85 & 1.19 & 0.00000061 & 0.0000008 & 0.000002 & 0.068 & 4059 & \\
\hline Variety & $0.00654^{* *}$ & 1.18 & $27.22^{* *}$ & $0.00000470^{* *}$ & $0.0000538^{* *}$ & $0.000091^{* *}$ & $0.536^{* *}$ & $3666537^{* *}$ & \\
\hline Foliar application & $0.01230^{* *}$ & $250.07^{*}$ & $74.76^{* *}$ & $0.00004808^{* *}$ & $0.0000273^{* *}$ & $0.000148^{* *}$ & $5.626^{* *}$ & $4385612^{* *}$ & \\
\hline Variety $\times$ stress & 0.00029 & 5.62 & $4.20^{* *}$ & 0.00000008 & $0.0000080^{* *}$ & $0.000008^{* *}$ & 0.129 & $45562^{*}$ & \\
\hline Foliar application $\times$ stress & $0.00087^{* *}$ & 65.18 & $7.40^{* *}$ & 0.00000030 & 0.0000002 & 0.000000 & 0.037 & $57885^{* *}$ & \\
\hline Variety $\times$ foliar application & 0.00012 & 72.29 & 1.37 & 0.00000026 & 0.0000011 & 0.000006 & 0.022 & $121523^{* *}$ & \\
\hline Variety $\times$ stress $\times$ foliar application & 0.00018 & 12.74 & 0.12 & 0.00000011 & 0.0000003 & 0.000006 & 0.027 & 12497 & \\
\hline Errorb & 0.00014 & 50.77 & 0.47 & 0.00000062 & 0.0000005 & 0.000001 & 0.056 & 9922 & \\
\hline $\mathrm{CV}(\%)$ & 2.491 & 7.7 & 3.47 & 4.45 & 2.3 & 2.12 & 5.3 & 3.6 & \\
\hline
\end{tabular}

Table 2. Mean comparison on yield, physiological traits and seed vigor of two soybean cultivars

\begin{tabular}{|c|c|c|c|c|c|c|}
\hline The main effects & $\begin{array}{l}\text { Soluble protein } \\
(\mathrm{mg} / \mathrm{g} / \mathrm{FW})\end{array}$ & $\begin{array}{c}\text { Superoxide } \\
\text { dismutase(\% inhibition) }\end{array}$ & $\begin{array}{c}\text { Peroxidase } \\
\text { (Abs mg-1 protein min-1) }\end{array}$ & $\begin{array}{c}\text { Catalase } \\
(\text { Absmg-1 protein min-1) }\end{array}$ & $\begin{array}{l}\text { Chlorophyll } a \\
(\mathrm{mgg}-1 \mathrm{FW})\end{array}$ & $\begin{array}{l}\text { Chlorophyll } b \\
\text { (mgg-1 FW) }\end{array}$ \\
\hline \multirow{3}{*}{ Stress } & $2.66 a$ & $4.52 \mathrm{~b}$ & $3.15 c$ & $132 \mathrm{c}$ & $1.41 \mathrm{a}$ & $0.73 a$ \\
\hline & $1.79 \mathrm{c}$ & $7.06 \mathrm{a}$ & $5.90 \mathrm{a}$ & $185 a$ & $0.75 c$ & $0.31 \mathrm{c}$ \\
\hline & $2.05 b$ & $6.98 \mathrm{a}$ & $5.70 \mathrm{~b}$ & $174 \mathrm{~b}$ & $1.01 \mathrm{~b}$ & $0.45 b$ \\
\hline \multirow{2}{*}{ Variety } & $2.11 \mathrm{~b}$ & $6.45 a$ & $4.74 b$ & $165 a$ & $0.99 \mathrm{~b}$ & $0.47 b$ \\
\hline & $2.24 \mathrm{a}$ & $5.92 b$ & $5.38 \mathrm{a}$ & $159 \mathrm{~b}$ & $1.12 \mathrm{a}$ & $0.52 \mathrm{a}$ \\
\hline \multirow{4}{*}{ Foliar application } & $2.07 \mathrm{~b}$ & $5.54 \mathrm{~b}$ & $5.79 \mathrm{a}$ & $174 a$ & $0.99 \mathrm{~b}$ & $0.44 \mathrm{~b}$ \\
\hline & $2.08 \mathrm{~b}$ & $5.52 b$ & $5.58 \mathrm{a}$ & $170 \mathrm{a}$ & $0.98 b$ & $0.44 \mathrm{~b}$ \\
\hline & $2.36 \mathrm{a}$ & $7.50 \mathrm{a}$ & $3.64 \mathrm{~b}$ & $143 \mathrm{~b}$ & $1.20 \mathrm{a}$ & $0.61 \mathrm{a}$ \\
\hline & $\begin{array}{l}\text { Total chlorophyll (mgg-1 } \\
\text { FW) }\end{array}$ & $\begin{array}{c}\text { Proline } \\
(\mathrm{mgg}-1 \mathrm{FW})\end{array}$ & $\begin{array}{l}\text { Solublecarbohydrates } \\
\qquad\left(\mu g^{-1} \mathrm{FW}\right)\end{array}$ & $\mathrm{fv} / \mathrm{fm}$ & Germination percentage & $\begin{array}{c}\text { Rate of } \\
\text { germination }\end{array}$ \\
\hline \multirow{3}{*}{ Stress } & $2.14 \mathrm{a}$ & $3.29 \mathrm{c}$ & $15963 c$ & $0.534 \mathrm{a}$ & $98.88 \mathrm{a}$ & $22.11 \mathrm{a}$ \\
\hline & $1.06 \mathrm{c}$ & $46.73 a$ & $21280 a$ & $0.422 \mathrm{c}$ & $93.33 b$ & $20.72 b$ \\
\hline & $1.45 \mathrm{~b}$ & $43.44 \mathrm{~b}$ & $21010 \mathrm{~b}$ & $0.426 b$ & $82.88 \mathrm{c}$ & $16.63 c$ \\
\hline \multirow{2}{*}{ Variety } & $1.47 \mathrm{~b}$ & $30.99 a$ & $19103 b$ & $0.454 \mathrm{~b}$ & $91.55 \mathrm{a}$ & $19.25 b$ \\
\hline & $1.64 \mathrm{a}$ & $31.32 \mathrm{a}$ & $19732 a$ & $0.484 \mathrm{a}$ & $91.85 \mathrm{a}$ & $20.38 a$ \\
\hline \multirow{4}{*}{ Foliar application } & $1.43 \mathrm{~b}$ & $44.37 \mathrm{a}$ & $20876 a$ & $0.457 b$ & $89.77 b$ & $18.71 \mathrm{a}$ \\
\hline & $1.41 \mathrm{~b}$ & $43.79 a$ & $20769 a$ & $0.460 \mathrm{~b}$ & $89.33 b$ & $18.58 \mathrm{a}$ \\
\hline & $1.81 \mathrm{a}$ & $5.29 \mathrm{~b}$ & $16608 b$ & $0.491 \mathrm{a}$ & $96.00 \mathrm{a}$ & $22.17 b$ \\
\hline & Radical dry weight (g) & Shoot dry weight (g) & Seedlingdry weight (g) & Seedlingvigour index & Grain yield (kg/ha) & \\
\hline \multirow{3}{*}{ Stress } & $0.0186 \mathrm{a}$ & $0.0315 \mathrm{a}$ & $0.0502 \mathrm{a}$ & $5.05 \mathrm{a}$ & $3246 a$ & \\
\hline & $0.0176 \mathrm{~b}$ & $0.0298 \mathrm{~b}$ & $0.0474 \mathrm{~b}$ & $4.48 \mathrm{~b}$ & $2611 b$ & \\
\hline & $0.0166 c$ & $0.0283 c$ & $0.0450 \mathrm{c}$ & $3.75 c$ & $2374 c$ & \\
\hline \multirow{2}{*}{ Variety } & $0.0179 \mathrm{a}$ & $0.0309 a$ & $0.0488 \mathrm{a}$ & $4.52 \mathrm{a}$ & $2483 b$ & \\
\hline & $0.0173 b$ & $0.0289 b$ & $0.0462 b$ & $4.32 \mathrm{~b}$ & $3004 a$ & \\
\hline \multirow{3}{*}{ Foliar application } & $0.0166 b$ & $0.0291 b$ & $0.0458 b$ & $4.14 \mathrm{~b}$ & $2470 \mathrm{~b}$ & \\
\hline & $0.0167 \mathrm{~b}$ & $0.0292 b$ & $0.0459 \mathrm{~b}$ & $4.07 \mathrm{~b}$ & $2448 b$ & \\
\hline & $0.0195 \mathrm{a}$ & $0.0313 \mathrm{a}$ & $0.0508 \mathrm{a}$ & $5.07 \mathrm{a}$ & $2314 \mathrm{a}$ & \\
\hline
\end{tabular}

Means in each column and for each treatment, followed by similar letter(s) are not significantly different

Kottapalli et al., 2009). In inadequate conditions, the pathway of proteins breakdown is open, because the plants use the proteins for the synthesis of nitrogen compounds as amino acids that might auxiliary influence the plant osmotic adjustment (Sankar et al., 2007). Similar results on proteins' reduction were found by Ramos et al. (1999) when investigating the effects of the water stress in Phaseolus vulgaris. The elevated protease activity under stressed conditions appeared to be part of an adaptive potential, since it led to the accumulation of free amino acids which contributed to the osmotic adjustment and was related to drought tolerance (Ramanjulu and Sudhakar, 1997). Crucial changes in water homeostasis bring on a decline in the chemical activity of water and loss of turgor in plant cells, which will disturb regular nitrogen metabolism such as protein degradation, and induce plant 
Table 3. Interaction effect of variety and water stress in yield physiological traits and seed vigor of two soybean cultivar

\begin{tabular}{|c|c|c|c|c|c|c|c|}
\hline Stress levels & & Soluble protein & Peroxidase & Catalase & Chlorophyll $a$ & Chlorophyll $b$ & Chlorophyll $a+b$ \\
\hline \multirow{2}{*}{$\mathrm{I}_{1}$} & $\mathrm{~V}_{1}$ & $2.70 \mathrm{a}$ & $3.24 \mathrm{a}$ & $130 \mathrm{a}$ & $1.39 \mathrm{~b}$ & $0.30 \mathrm{~b}$ & $2.09 \mathrm{~b}$ \\
\hline & $\mathrm{V}_{2}$ & $2.82 \mathrm{~b}$ & $3.06 \mathrm{~b}$ & $133 \mathrm{a}$ & $1.44 \mathrm{a}$ & $0.32 \mathrm{a}$ & $2.20 \mathrm{a}$ \\
\hline \multirow{2}{*}{$\mathrm{I}_{2}$} & $\mathrm{~V}_{1}$ & $1.65 \mathrm{~b}$ & $4.63 \mathrm{a}$ & $190 \mathrm{a}$ & $0.67 b$ & $0.41 \mathrm{~b}$ & $1.96 \mathrm{~b}$ \\
\hline & $\mathrm{V}_{2}$ & $1.20 \mathrm{a}$ & $4.29 \mathrm{~b}$ & $179 \mathrm{~b}$ & $0.84 \mathrm{a}$ & $0.48 \mathrm{a}$ & $1.16 \mathrm{a}$ \\
\hline \multirow{3}{*}{$\mathrm{I}_{3}$} & $\mathrm{~V}_{1}$ & $1.72 \mathrm{~b}$ & $4.59 \mathrm{a}$ & $175 \mathrm{a}$ & $0.94 \mathrm{~b}$ & $0.70 \mathrm{~b}$ & $1.35 \mathrm{~b}$ \\
\hline & $\mathrm{V}_{2}$ & $2.23 \mathrm{a}$ & $4.25 \mathrm{~b}$ & $165 \mathrm{~b}$ & $1.07 \mathrm{a}$ & $0.75 a$ & $1.56 \mathrm{a}$ \\
\hline & & $\begin{array}{c}\text { Soluble } \\
\text { carbohydrates }\end{array}$ & $\begin{array}{c}\text { Rate of } \\
\text { germination }\end{array}$ & $\begin{array}{c}\text { Shoot dry } \\
\text { weight }\end{array}$ & Seedling dry weight & Grain yield & \\
\hline \multirow{2}{*}{$\mathrm{I}_{1}$} & $V_{1}$ & $15862 \mathrm{~b}$ & $20.98 \mathrm{~b}$ & $0.0318 \mathrm{a}$ & $0.0507 \mathrm{a}$ & $3018 \mathrm{~b}$ & \\
\hline & $\mathrm{V}_{2}$ & $16065 \mathrm{a}$ & $23.22 \mathrm{a}$ & $0.0313 \mathrm{a}$ & $0.0497 \mathrm{~b}$ & $3474 a$ & \\
\hline \multirow{2}{*}{$\mathrm{I}_{2}$} & $V_{1}$ & $20868 \mathrm{~b}$ & $20.37 a$ & $0.0311 \mathrm{a}$ & $0.0491 \mathrm{a}$ & $2285 \mathrm{~b}$ & \\
\hline & $\mathrm{V}_{2}$ & $21692 a$ & $21.06 \mathrm{a}$ & $0.0286 \mathrm{~b}$ & $0.0458 \mathrm{~b}$ & $2937 a$ & \\
\hline \multirow{2}{*}{$\mathrm{I}_{3}$} & $\mathrm{~V}_{1}$ & $20580 \mathrm{~b}$ & $16.39 a$ & $0.0299 a$ & $0.0468 \mathrm{a}$ & $2146 b$ & \\
\hline & $\mathrm{V}_{2}$ & $21439 a$ & $16.85 \mathrm{a}$ & $0.0269 \mathrm{~b}$ & $0.0433 \mathrm{~b}$ & $2602 \mathrm{a}$ & \\
\hline
\end{tabular}

At each stress level, surface treatments with the same letters are shown, with no significant difference.

$\mathrm{I}_{1}=$ well watering or control treatment, $\mathrm{I}_{2}=$ stressed by skipping the irrigation at vegetative stage until $10 \%$ flowering $\left(\mathrm{V}_{5}-\mathrm{R}_{1}\right), \mathrm{I}_{3}=$ irrigation disruption during flowering and $10 \%$ poding growth stages $\left.\left(\mathrm{R}_{1}-\mathrm{R}_{3}\right)\right] ; \mathrm{V}_{1}=$ 'L17', $\mathrm{V}_{2}=$ 'Clark63'; $\mathrm{F}_{0}=$ no application, $\mathrm{F}_{1}=$ foliar application of distilled water and $\mathrm{F}_{2}$ of zinc sulfate.

Table 4. Interaction effect of water stress and foliar application in yield physiological traits and seed vigor of two soybean cultivars

\begin{tabular}{|c|c|c|c|c|c|c|c|}
\hline $\begin{array}{l}\text { Stress } \\
\text { level }\end{array}$ & Foliar application levels & $\begin{array}{l}\text { Superoxide } \\
\text { dismutase }\end{array}$ & Peroxidase & Catalase & Chlorophyll $a$ & Chlorophyll $b$ & Chlorophyll $a+b$ \\
\hline \multirow{3}{*}{$\mathrm{I}_{1}$} & $\mathrm{~F}_{0}$ & $3.62 \mathrm{~b}$ & $3.32 \mathrm{a}$ & $136 \mathrm{a}$ & $1.38 \mathrm{~b}$ & $0.68 \mathrm{~b}$ & $2.10 \mathrm{~b}$ \\
\hline & $\mathrm{F}_{1}$ & $3.63 \mathrm{~b}$ & $3.23 \mathrm{a}$ & $136 \mathrm{a}$ & $1.35 \mathrm{~b}$ & $0.69 \mathrm{~b}$ & $2.05 \mathrm{~b}$ \\
\hline & $\mathrm{F}_{2}$ & $6.30 \mathrm{a}$ & $2.90 \mathrm{~b}$ & $124 \mathrm{~b}$ & $1.51 \mathrm{a}$ & $0.81 \mathrm{a}$ & $2.32 \mathrm{a}$ \\
\hline \multirow{3}{*}{$\mathrm{I}_{2}$} & $\mathrm{~F}_{0}$ & $6.55 \mathrm{~b}$ & $7.34 \mathrm{a}$ & $185 \mathrm{a}$ & $0.71 b$ & $0.25 b$ & $0.96 \mathrm{~b}$ \\
\hline & $\mathrm{F}_{1}$ & $6.56 \mathrm{~b}$ & $7.35 \mathrm{a}$ & $182 \mathrm{a}$ & $0.69 \mathrm{~b}$ & $0.25 b$ & $0.93 \mathrm{~b}$ \\
\hline & $\mathrm{F}_{2}$ & $8.05 \mathrm{a}$ & $4.33 \mathrm{~b}$ & $150 \mathrm{~b}$ & $0.86 \mathrm{a}$ & $0.43 \mathrm{a}$ & $1.30 \mathrm{a}$ \\
\hline \multirow{3}{*}{$\mathrm{I}_{3}$} & $\mathrm{~F}_{0}$ & $6.44 b$ & $6.72 \mathrm{a}$ & $200 \mathrm{a}$ & $0.89 \mathrm{~b}$ & $0.38 \mathrm{~b}$ & $1.27 \mathrm{~b}$ \\
\hline & $\mathrm{F}_{1}$ & $6.37 \mathrm{~b}$ & $6.70 \mathrm{a}$ & $198 \mathrm{a}$ & $0.89 \mathrm{~b}$ & $0.37 \mathrm{~b}$ & $1.26 \mathrm{~b}$ \\
\hline & $\mathrm{F}_{2}$ & $8.14 \mathrm{a}$ & $3.68 \mathrm{~b}$ & $170 \mathrm{~b}$ & $1.24 \mathrm{a}$ & $0.59 \mathrm{a}$ & $1.83 \mathrm{a}$ \\
\hline $\begin{array}{l}\text { Stress } \\
\text { level }\end{array}$ & Foliar application levels & Proline & $\begin{array}{c}\text { Soluble } \\
\text { carbohydrates }\end{array}$ & $\mathrm{fv} / \mathrm{fm}$ & $\begin{array}{c}\text { Rate of } \\
\text { germination }\end{array}$ & Grain yield & \\
\hline \multirow{3}{*}{$\mathrm{I}_{1}$} & $\mathrm{~F}_{0}$ & $3.68 \mathrm{a}$ & $16529 \mathrm{a}$ & $0.529 \mathrm{~b}$ & $21.31 \mathrm{~b}$ & $3106 \mathrm{~b}$ & \\
\hline & $\mathrm{F}_{1}$ & $3.41 \mathrm{a}$ & $16416 a$ & $0.530 \mathrm{~b}$ & $21.38 \mathrm{~b}$ & $2989 \mathrm{~b}$ & \\
\hline & $\mathrm{F}_{2}$ & $2.77 \mathrm{~b}$ & $14945 \mathrm{~b}$ & $0.540 \mathrm{a}$ & $23.62 \mathrm{a}$ & $3644 a$ & \\
\hline \multirow{3}{*}{$\mathrm{I}_{2}$} & $\mathrm{~F}_{0}$ & $67.12 \mathrm{a}$ & $23176 a$ & $0.389 \mathrm{~b}$ & $19.86 \mathrm{~b}$ & $2295 \mathrm{~b}$ & \\
\hline & $\mathrm{F}_{1}$ & $66.12 \mathrm{a}$ & $23066 \mathrm{a}$ & $0.388 \mathrm{~b}$ & $19.85 \mathrm{~b}$ & $2325 \mathrm{~b}$ & \\
\hline & $\mathrm{F}_{2}$ & $6.94 b$ & $17597 \mathrm{~b}$ & $0.430 \mathrm{a}$ & $22.44 \mathrm{a}$ & $3215 a$ & \\
\hline \multirow{3}{*}{$\mathrm{I}_{3}$} & $\mathrm{~F}_{0}$ & $62.31 \mathrm{a}$ & $22922 \mathrm{a}$ & $0.452 \mathrm{~b}$ & $14.49 \mathrm{~b}$ & $2011 b$ & \\
\hline & $\mathrm{F}_{1}$ & $61.85 \mathrm{a}$ & $22824 \mathrm{a}$ & $0.460 \mathrm{~b}$ & $14.94 \mathrm{~b}$ & $2030 \mathrm{~b}$ & \\
\hline & $\mathrm{F}_{2}$ & $6.17 \mathrm{~b}$ & $17283 \mathrm{~b}$ & $0.509 \mathrm{a}$ & $20.44 \mathrm{a}$ & $3082 a$ & \\
\hline
\end{tabular}

At each stress level, surface treatments with the same letters are shown, with no significant difference.

arrest, or death finally (Hsiao, 1973); consequently, it can be hypothesized that increased leaf protein contents is relevant to modifications in plant water relations through Zn foliar modulation.

Lower protein concentration in leaves of both tested cultivars demonstrated the role of $\mathrm{Zn}$ in protein synthesis, which in line with Gadallah (2000) who reported enhanced soluble protein accumulation and decreased total free amino acids in shoots of soybean (Glycine max cv. 'Klark') under severe drought stress (-1.5 MPa) due to $\mathrm{Zn}$ supplementation. Marschner (1995) noted that $\mathrm{Zn}$ played a fundamental role in several critical cellular functions including protein metabolism and gene expression. In common bean (Cakmak et al., 1989) or pistachio (Tavallali et al., 2010) plants suffering from $\mathrm{Zn}$ deficiency, protein synthesis has been also impaired.

Analysis of the activity of antioxidant enzymes SOD, CAT and POD revealed that skipping one irrigation treatment at any growth phases lead to an increase in their activity (Table 2). Drying conditions induced higher activity of CAT and POD in 'L17' (Table 3). CAT activity did not differ between well-watered leaves of both the soybean cultivars. However, POD and total leaf protein was significantly higher in 'L17' than that of 'Clark63' (Table 3). Osmotic stress can result in increased concentrations of AOS, therefore AOS-scavenging antioxidant enzymes, such as SOD and CAT play a vital role in removing these destructive oxidant species. Excess production of toxic $\mathrm{O}_{2}$ species during water deficit stress results from impaired electron transport systems such as chloroplasts and mitochondria, as well as via other pathways such as photorespiration, by catalyzing the detoxification of $\mathrm{O}_{2}^{-}$to $\mathrm{H}_{2} \mathrm{O}_{2}$ and $\mathrm{O}_{2}$, SOD blocks cell damage caused by $\mathrm{O}_{2}^{-} \cdot \mathrm{H}_{2} \mathrm{O}_{2}$ is also toxic to cells and has to be further detoxified by CAT and/or peroxidases to water and $\mathrm{O}_{2}$.

Increased SOD activity under imposed oxidative drought stress (Table 2) was similar to the findings of Luna and et al. (1985). Variations in SOD activity have been previously reported depending on drought severity, duration and species (Luna et al., 1985). Both of the hereby studied cultivars had different SOD activity (Table 2 ), with significant differences in the case of antioxidant enzymes activity. Lower activity of SOD in 'Clark63' was pertinent to the notion that there may be no increased formation of $\mathrm{O}_{2}$ in chloroplasts of this cultivar (Table 2) 
186

since this $\mathrm{O}_{2}$ processing enzyme is known to be substrateinducible. Overproduction of hydrogen peroxides as a resultant of higher SOD activity stimulated $\mathrm{H}_{2} \mathrm{O}_{2}$ metabolizing enzyme-CAT- in L17 leaves (Table 3). Confirming the current results, Turkan et al. (2005) reported lower sensitivity of SOD and significant increased constitutive levels of catalase and ascorbate peroxidase in drought-tolerant Phaseolus acutifolius L. species, compared with those of drought-sensitive $P$. vulgaris, under water stress mediated using polyethylene glycol. It may be concluded that higher constitutive levels of $P$. acutifolius suggested a more effective $\mathrm{H}_{2} \mathrm{O}_{2}$ dismutation capacity outside the chloroplasts under osmotic stress.

Under all irrigation treatments, CAT and POD activity decreased by foliar $\mathrm{Zn}$ application; conversely, levels of SOD activity enhanced profoundly due to adequate $\mathrm{Zn}$ supply, particularly in plants grown under favorable soil moisture condition (Table 4). Furthermore, the two cultivars exhibited different responses to $\mathrm{Zn}$ treatment in this regard. The incensement in SOD activity was larger for 'Clark63' (Table 5). In several studies, decreased SODs activity in $\mathrm{Zn}$-deprived leaves has been documented (Cakmak and Marshner, 1993; Wang and Jin, 2007; Tavallali et al., 2010). Among SOD isoform, $\mathrm{Cu}-\mathrm{ZnSOD}$ has a predominant role in scavenging $\mathrm{O}_{2}$, and thus $\mathrm{Zn}$ participates in enzymatic defense of cells against free radicals (Fridrovich, 1986).

In $\mathrm{Zn}$-deficient leaves of common bean (Cakmak and Marschner, 1993), pistachio (Tavallali et al., 2010), maize (Wang and Jin, 2007) and wheat (Yilmaz et al., 1998), CAT and POD activity has been reported, but in the present study converse results were observed (Table 2). Wang and Jin (2007) reported that in maize seedlings $\mathrm{Zn}$ supply had little and negligible effect on activation of CAT. The reduced activity levels of CAT and POD in Zn-treated soybean plants could attribute to improved root growth as a result of supplementary $\mathrm{Zn}$ application (Gadallah, 2000), thus leading to increase the crop ability to use the moisture reserves in the soil, and thereby plants will be able to have higher water retaining capacity. It seems that under such conditions, free radicals production, including $\mathrm{H}_{2} \mathrm{O}_{2}$, due to drought stress, would be less intense, which in turn leads to a decrease in some antioxidant enzymes.

Peroxideses have various physiological roles in plant cells and participate in many reactions including lignifications, cross linking of cell elongation and phenol oxidation. Peroxidase activity shows a close correlation with changes in physiological processes such as respiration, photosynthesis and transpiration and therefore has the potential to serve as a sensitive indicator of comprised metabolic activity (Verkleij and Schat, 1990). From the results of the current trial, it could be hypothesized that POD may be involved in internal detoxification mechanisms of soybean leaves against zinc deficiency. It was clear, as shown in Table 2, that CAT and POD activities during reproductive period was significantly less compared with the vegetative one.

As plant response is triggered by a stress factor, the activities of the antioxidant enzymes increased in the initial stage of the stress, providing a certain degree of protection from oxidative damage, and then decline with the duration of the stress, due to either reduced synthesis or enhanced degradation or inactivation of the enzymes or all of these factors. An increase in SOD and CAT activities followed by a decrease in response to drought was recorded at the initial stage of the stress in wheat (Zhang and Kirkham, 1994).

It is important to note that irrigation disruption during vegetative and reproductive stages or normal irrigation $\times$ foliar application of distilled water treatment had the same values for above-mentioned biochemical indices (Table 4). Similar results were obtained by Thomas et al. (2004). Such differences might be due to the variations existing among plant nutrients roles in stress resistance and repair mechanisms (Michauil et al., 2004).

\section{Chlorophyll concentrations}

Total chlorophyll, as well as chlorophyll $a$ and chlorophyll $b$ contents decreased extensively in response to water and zinc deficiency stresses (Table 2). Decreased or unchanged chlorophyll level during drought stress has been reported in several species, depending on the duration and severity of drought (Zhang and Kirkham, 1996). On the other hand, imposed drought stress during vegetation period affected biosynthesis and consequently chl content more negatively (Table 2), which can be relevant to the higher sensitivity of crop to available soil moisture at this developmental phase. Considerable reduction in chl content due to water stress was reported in most crop species (Ashraf et al., 1994) mainly because of the damages of overproduced free radicals to chloroplast fine structures (Smirnoff, 1995). Furthermore, water deficit induced reduction in chlorophyll content has been ascribed to loss of chloroplast membranes, excessive swelling, distortion of the lamellae vesiculation, and the appearance of lipid droplets (Garg et al., 1998). Low concentrations of photosynthetic pigments can directly limit the photosynthetic potential and hence primary production. Both chlorophyll $a$ and $b$ are prone to soil dehydration.

Drought stressed leaves of 'L17' had lower chlorophyll $a$, chlorophyll $b$ and total chlorophyll concentrations when compared to 'Clark63' and the differences manifested intensely at R1-R3 drying conditions (Table 3). It seemed that the ability of 'Clark63' in maintenance of a high chlorophyll content under water deficit stress was resulted from a more efficient antioxidative system as well as higher leaf soluble carbohydrates (Table 2), which could contribute in improving leaves water status. Thereby, detrimental consequences of oxidative drought stress on biomembranes, including thylacoid membranes, would probably decrease in 'Clark63'. Confirming this result, Turkan et al. (2005) reported that in Phaseolus acutifolius L. the direct consequence of higher relative leaf water content was a more efficient activity of some antioxidant enzymes such as CAT. The decrease in leaf chl content of common bean drought-tolerant cultivars with high catalase and ascorbate peroxidase activities was lower than those of susceptible genotypes. Total chlorophyll content of 'Clark63' plants treated with distilled water or with $\mathrm{Zn}$ was significantly higher as compared with $\mathrm{F}_{0}$ (Table 5) and the differences were exacerbated by $\mathrm{Zn}$ foliar application. 
The application of exogenous zinc to leaves of soybean plants induced the accumulation of chlorophyll content of leaves (Table 2). Among the three watering regimes, the magnitude of increase in chlorophyll biosynthesis and photosynthetic pigment contents due to $\mathrm{Zn}$ supplementation through foliar application was higher for $\mathrm{I}_{2}$ treatment (Table 4). Zinc is involved in chlorophyll formation by taking part in the regulation of cytoplasmic concentrations of nutrients (Fischer, 1997). The loss of photosynthetic pigments and the development of symptoms like chlorosis and necrosis in plants receiving $\mathrm{Zn}$ at inadequate levels may be possibly due to enhanced AOS production (Cakmak, 1994), as showed in higher CAT and POD activity, implying that $\mathrm{Zn}$ deprivation is accompanied by over accumulation of toxic $\mathrm{O}_{2}$ such as hydrogen peroxides in $\mathrm{Zn}$ deficient leaves. The increased generation $\mathrm{O}_{2}^{-}$in $\mathrm{Zn}$-deficient plants is likely resulting from the decrease availability of reductants, leading to an accumulation of high-potential electrons and excited chlorophyll molecules (Grossman and Takhash, 2001). Effect of distilled water application on leaf chlorophyll concentrations was not notable at any irrigation treatment (Table 4).

\section{Proline accumulation}

Free proline levels were significantly affected by the irrigation levels and different foliar application treatments (Table 1). The proline content of leaves of both cultivars was almost the same (Table 2). Water deficit imposed at two developmental stages caused a marked augmentation of proline in leaves. However, the impact of drought stress on this physiological index was more evident when stress was exerted at vegetative phase (Table 2), which might be an indicator of higher drought intensity to soybean plants submitted to water deficits during this stage. The accumulation of certain cytosolutes, particularly proline, should avoid the detrimental effects of a low osmotic potential of the cells, without interfering with protein synthesis. The duration and intensity of stress, as well as plant species and genotype, are the factors impressing proline aggregation (Ragi, 1994). Accumulation of proline is an important indicator of drought stress tolerance in bacteria, algae and higher plants (Aspinall and Paleg, 1981). This amino acid has been reported to play multiple physiological functions in plants subjected to abiotic stress conditions, such as stabilizer of macromolecules, osmoregulation, a sink for excess reductant, a store of carbon and nitrogen for use after stress factor relief, and a signal of senescence (Aspinall and Paleg, 1981). As shown in Table 2, high proline content was associated with protein hydrolysis and reduced total leaf protein content induced by water deficit. Confirming this results, Yadav et al. (1999) found that increased amino acid content, particularly proline, under drought stress was obviously due to hydrolysis of proteins in chickpea (Cicer arietinum L.) leaves.

Application of $\mathrm{Zn}$ to the foliage of soybean cultivars had a drastic negative effect on leaf free proline levels as compared with $\mathrm{F}_{0}$ and $\mathrm{F}_{1}$ treatments (Table 2). Such a decline was observed under all three irrigation regimes, particularly when sever stress was noted (Table 4). Thalooth et al. (2006) observed no clear effect of zinc or magnesium foliar application on mungbean leaf proline aggregation. On the other hand, proline content in ' $\mathrm{L} 17$ ' leaves was less modified by $\mathrm{Zn}$ supply relative to 'Clark63' (Table 2). There was no distinct effect of distilled water application on the proline biosynthesis and augmentation, as compared with $\mathrm{F}_{0}$ (Table 4).

It was demonstrated that glutamate is the precursor of proline and chlorophyll in higher plants (Savitskaya, 1976). It seems that when plants received adequate $\mathrm{Zn}$ through foliar application, majority part of glutamate participate in chl biosynthesis, that cause to decline input of this compound to proline biosynthesis pathway.

\section{Total soluble carbohydrates}

Variance analysis results presented in Table 2 revealed that irrigation disruption at any soybean growth stage augmented total leaf sugar content, relative to full irrigation treatment, especially when plants experienced soil drought conditions at vegetative stage. This was possibly resulted from the coincidence of vegetative period of soybean cultivars with higher temperature of experimental area, which impressed synergistically the crop, and caused the highest sugar production of shoots. Higher concentrations of soluble carbohydrate were employed by 'Clark63' under different drought conditions (Table 3).

Plants accumulate different types of organic and inorganic solutes in the cytosol to lower osmotic potential, thereby maintaining cell turgor (Mullet and Whitsitt, 1996). The maintenance of leaf turgor may be achieved by osmotic adjustment in response to the accumulation of proline, sucrose, soluble carbohydrates, glycinebetaine and other solutes in cytoplasm, improving water uptake from drying soil and increase the crop resistance under hostile environmental conditions such as drought (Crowe et al., 1988).

Sprayed leaves with $\mathrm{Zn}$ had noticeably lower soluble sugar than those treated with distilled water or which have not been treated (Table 2). This trend was observed under all watering regimes (Table 4). The sugar content of leaves in $F_{0}$ and $F_{1}$ treatments was statistically similar (Table 4). Furthermore, in 'Clark63' total leaf carbohydrates reduction was higher than that of ' $\mathrm{L} 17$ ', as a result of $\mathrm{Zn}$ addition (Table 5). These observations are parallel with those obtained by Gadallah (2000), who found that enhanced soluble sugars accumulation had happened in roots of soybean plants, not in the leaves, when supplied with zinc.

\section{The potential yield of photosystem II (PSII)}

The maximum quantum yield of PSII photochemistry, represented by $\mathrm{Fv} / \mathrm{Fm}$ ratio, was inhibited by water - and $\mathrm{Zn}$ deficiency stresses (Table 2). The electron transport capacity was more hampered due to water shortage during vegetative stage (Table 2), thus leading to low leaf chlorophyll concentrations

Table 5. Interaction effect of variety and foliar application in yield and physiological traits of two soybean cultivars

\begin{tabular}{lccccc}
\hline Stress levels & & Superoxide dismutase & Total chlorophyll & Proline & Soluble carbohydrates \\
\hline \multirow{2}{*}{$\mathrm{F}_{0}$} & $\mathrm{~V}_{1}$ & $5.95 \mathrm{a}$ & $1.36 \mathrm{~b}$ & $43.46 \mathrm{~b}$ & $20519 \mathrm{~b}$ \\
& $\mathrm{~V}_{2}$ & $5.13 \mathrm{~b}$ & $1.50 \mathrm{a}$ & $45.28 \mathrm{a}$ & $21233 \mathrm{a}$ \\
$\mathrm{F}_{1}$ & $\mathrm{~V}_{1}$ & $5.90 \mathrm{a}$ & $1.33 \mathrm{~b}$ & $43.63 \mathrm{a}$ & $20376 \mathrm{~b}$ \\
& $\mathrm{~V}_{2}$ & $5.14 \mathrm{~b}$ & $1.49 \mathrm{a}$ & $43.96 \mathrm{a}$ & $21161 \mathrm{a}$ \\
$\mathrm{F}_{2}$ & $\mathrm{~V}_{1}$ & $7.51 \mathrm{a}$ & $1.71 \mathrm{~b}$ & $5.87 \mathrm{a}$ & $2743 \mathrm{a}$ \\
& $\mathrm{V}_{2}$ & $7.48 \mathrm{a}$ & $1.92 \mathrm{a}$ & $4.71 \mathrm{~b}$ & $2751 \mathrm{~b}$ \\
\hline
\end{tabular}

At each stress level, surface treatments with the same letters are shown, with no significant difference. 
188

(Table 2). Between the two cultivars, 'Clark63' was superior in the case of this trait.

Chlorophyll fluorescence measurement has become a widely used method to study the functioning of the photosynthetic apparatus and is a sensitive indicator of changes in thylakoid membrane integrity caused by environmental stresses (Bukhov, 2004). Despite the fact that PSII is highly drought resistant (Yordanov et al., 2003), under water stress, photosynthetic electron transport through PSII is inhibited. Several in vivo studies demonstrated that water deficit results in damages of the PSII oxygen-evolving complex (Lu and Zhang, 1999) and of the PSII reaction centers associated with the degradation of $\mathrm{D}_{1}$ protein (Cornic and Briantais, 1991). We came into the same results, implying that electron transport capacity in soybean plants was not resilient to imposed drought stress intensity regardless of plant $\mathrm{Zn}$ nutrition.

Foliar Zn application substantially increased PSII activity (Table 2). Inhibitory effects of drought stress on PSII photochemistry was equally ameliorated by zinc nutrition at both growth stages, as compared with the other combinations (Table 3). Intensified chl florescence in $\mathrm{Zn}$-deficient leaves could be ascribing to impairment of free radicals scavenging systems (decreased SOD activity). As SODs have critical effect in antioxidative systems of all biological tissues (Bowler et al., 1992), it can be suggested that in plants with reduced SOD activity (including Zn deficiency) photosynthetic machinery should be highly sensitive to stress factors such as drought conditions. Depressed PSII photochemical activity because of $\mathrm{Zn}$ deprivation has been previously reported (Wang, et al., 2009).

\section{Seed quality}

Comparison means of main effects of the experimental factors reflected significant differences regarding germination percentage (GP), mean germination rate (GR), dry weight of radicles (RDW), shoots (SHDW), seedling (SDW) and seedling vigor index (SVI); the exception was for percentage of germination, which was not affected by cultivar (Table 1). Withholding irrigation during V5-R1 or R1-R3 stages, negatively affected all the above-mentioned seed qualitative parameters. Seeds produced from plants subjecting to drought stress at reproductive stage $\left(\mathrm{R}_{1}-\mathrm{R}_{3}\right)$ had obviously lower quality (Table 2), implying that in soybean, vegetative growth stage and early flowering are the developmental stages most tolerant to drought. This result is in accordance with Rassini and Lin (1981) who reported reductions in soybean seed vigor as drought stress increased during growth stages $R_{3}$ to $R_{5}$.

Unlike water shortage, seeds from plants sprayed with $\mathrm{Zn}$ indicated higher GR than those of $F_{0}$ and $F_{1}$. Significant interaction of irrigation versus cultivar and foliar treatments was observed for GR and SHDW (Table 3). When the soil moisture was sufficient, seeds of 'Clark63' were superior in GR; however, under water deficit treatments this trait was statistically similar in both cultivars under study. 'L17' had higher SDW, while seeds of 'Clark63' germinated faster (Table 2). This can be attributed to more zinc accumulation in seeds of 'Clark63' in all watering treatments that in turn could increase seed protein concentrations (Singh et al., 1992). Considering that proteins compounds contribute in water up take by seeds, thus cause progress inhibitions phases faster and subsequently enhanced seed germination. Shoot DW produced from 'Clark63' seeds was larger effected by water stress, while the difference between cultivars under normal irrigation regime was negligible (Table 3).
The effect of $Z n$ nutritional status of mother plants on produced seed quality when zinc supplementation was performed at soybean reproductive phase was more than that of vegetative growth period or favourable watering level (Table 4).

Water limitation during seed development usually interrupts the process and results in small seed size (Cruz-Aguado et al., 2000). The reduction in seed size is primarily due to a shortening of the filling period rather than an inhibition of seed growth rate (Vieira et al., 1992). Westgate et al. (1989) suggested that the moisture status of seeds during development is not affected by changes in plant water status and the rate of seeds dry matter accumulation was not affected by drought stress that later caused significant reductions in yield. A highly impressionable seed vigor from irrigation disruption during reproductive growth stage in comparison to seed quality obtained from soybean plants experienced imposed drought stress at vegetative period (Table 2) was noted, indicating that crop water requirement should be supplied during R1-R3 growth stages in order to produce high quality seed lots of soybean cultivars under water-limited circumstances.

Positive effects of increasing of some micronutrients amounts in seeds on germination, emergence and seedling growth were reported (Bonilla et al., 2004). In the current experiment it was documented that foliage treatment of soybean plants with $\mathrm{Zn}$ increased seed $\mathrm{Zn}$ content in parallel with enhanced seed germination and heavier seedling production of such seed lots (Table 2). These results coincide with those obtained on safflower (Carthamus tinctorius L.) by MovaheddyDehnavy et al. (2009). Beside the importance of nutritious quality of harvestable grain legumes for human diet (Graham $e t$ al., 1997), it is conceivable that the observed increase in seed quality of soybean through correcting the crop $\mathrm{Zn}$ deficiencies, resulted in standard establishment (higher germination rate) that is a critical factor to crop production under arid and rainfed agricultural systems (Bort and et al., 1998). Also, it can be postulated that soybean plants grown from high $\mathrm{Zn}$ seeds produced more root and shoot biomass, enabling the plants to take up and access other resources in later growth stages, as reported by Graham and Rengel (1993). Lowered mean germination of Zn-rich soybean seeds, relative to low-Zn seeds were collected from plants under $\mathrm{F}_{0}$ and $\mathrm{F}_{1}$ treatments (with $\mathrm{Zn}$ concentrations of $37.4 \mathrm{mg} \mathrm{kg}^{-1}$ seed), probably due to their larger size (data have not been shown) that delay progression of imbibition phases and increased mean time to radical protrusion. It is important to note that different sizes of seeds, having different levels of reserves and food storage is among the factors that influence seed vigor (Perry, 1980). Therefore, it seemed reasonable that heavier seeds from $\mathrm{Zn}$ treated plants exhibit higher quality (vigor and viability). The review by Cakmak (2008) provides further reasons for profits of $\mathrm{Zn}$-rich seeds on plant growth.

\section{Economical yield}

The main effects of factors were statistically significant on grain yield (Table 1). When submitted to water stress at different growth stages, grain yield was reduced by to 20 and $27 \%$, respectively (Table 2). However, the differential response of cultivars was observed with regard to yield production. Yield losses of 'L17' when water stress was exerted at both stages were greater, most clearly during vegetative stage until $10 \%$ flowering (Table 3). This may be associated with lasted growth cycle and later flowering of 'Clark63' and therefore, less vulnerability to 
decreasing soil moisture. Generally, 'Clark63' yielded better than 'L17' (Table 3). The obtained results evidenced that soybean cultivars are more susceptible to soils' dry periods at R1-R3 stage (Table 3). In legumes, the reproductive stage in the most sensitive stage to drought stress, whether it takes place during flowering formation (Pedroza and Munoz, 1993), or pod formation (Castaneda et al., 2006). This is because the water deficits cause falling or abortion of reproductive structures due to irreversibly arresting their development (Zinselmeier et al., 1995), as it occurred with the pistil in soybean (Kokubun et al., 2001) and pollen in dry bean (Shen and Webster, 1986) and which resulted in lower numbers of pod per plant (Boutra and Sanders, 2001); these relates in part to the reduction of photosynthesis and limited source flux from leaves to flowers and new-formed pods (Schussler and Westgat, 1995).

Grain yield per unit area raised to a great extent through $\mathrm{Zn}$ application over distilled water treatment or control (Table 2). The effect differed under various watering regimes (Table 4). As foliage $\mathrm{Zn}$ treatment under full irrigation was applied, water stress imposed at V5-R1 and R1-R3 led to an increment in grain yield up to 17,40 and $53 \%$ respectively. As well, positive effects of Zn nutrition on yield of 'L17' was more pronounced (Table 5). Morphological characteristics like greater leaf area of 'L17' could take part in such superiority by increased absorption surface of shoot and thus enhanced foliar applied fertilization advantages. Accelerating of growth, biomass production and yield attributes are possibly resultants of $\mathrm{Zn}$ roles in increased metabolism, biosynthesis of auxins, as well as a better uptake of other essential nutrients (Cakmak and Engels, 1999). This was ascribed to higher foliage and vegetative growth and accumulation of dry matter, more photosynthetic supply to root and also better utilization capacity of available nutrients by the soybean.

The beneficial role of $\mathrm{Zn}$ on ameliorating drought stress on yield has been acclaimed in previous studies (Khan et al., 2003; Tolooth et al., 2006; Movaheddy-Dehnavy et al., 2009). Apart from $\mathrm{Zn}$ effect on plants' water status (Gadallah, 2000), Zn nutrition can affect their susceptibility to drought-induced oxidative stress because of a protective role of zinc against photooxidative stress (Cakmak, 2000). It is therefore likely that drought stress-related production of ROS and sensitivity of plants to photoxidative damage in chloroplasts and yielding are additionally accentuated when plants would simultaneously suffer from Zn-deficiency stress, as shown in Table 2.

Another possible reason for yield rise through $\mathrm{Zn}$ addition could refer to photosynthesis process. Decline in photosynthetic $\mathrm{CO}_{2}$ fixation of $\mathrm{Zn}$-deficient leaves had been reported by several researchers. Limited stomatal aperture (Sharma et al., 1995) or carbonic anhydrase activity (Hatch and Slack, 1970), impaired phloem export of carbohydrates or decreased sink demand (Kitagishi and Obata, 1986), or impaired photosynthesis light stage, are the main factors that can reduce photosynthetic capacity in such leaves. Keeping in view the above facts, contribution of adequate $\mathrm{Zn}$ supply for improving yield of soybean plants grown under moisture deficit soil, could ascribe to enhancement of photosynthetic capacity by increasing or regulating of photo-assimilate synthesis, through raising photosynthetic pigments concentrations (chlorophylls $a$ and $b$ ) and PSII quantum yield (Table 2) and therefore, probably transporting to the sinks, under such conditions.

\section{Conclusions}

The current results pointed out the need for improvement of zinc nutritional status of soybean in regard to the maintenance and alleviation of inhibitory effects of drought stress on crop productivity under marginal environmental conditions (e.g. drought), specifically during initial flowering and pod formation. This adaptation resulted from significantly higher values of SODs activity, photosynthetic pigments $(\mathrm{chl} a$, chl $b$ and total chl) and electron transport efficiency through PSII, which ultimately proved beneficial for the photosynthetic machinery of the plant system under aggressive drought stress. It can be concluded that $\mathrm{Zn}$ deficiency stress represented an oxidative stress and promoted free radicals species production, since $\mathrm{Zn}$-deficient leaves, by hyping catalase and peroxides activity, defended themselves against zinc deficiency. Discernible rise of the activity of these enzymes in leaf suffering from $\mathrm{Zn}$ deficiency indicated hastened photo-oxidative damages. Irrespective of $\mathrm{Zn}$ application, 'Clark63' was a highyielding cultivar under both full water supply and limited watering regimes. Significantly higher accumulation of osmotically active solutes, as well as soluble protein contributed to 'Clark63' superiority in grain yield production, as they act against oxidative damage. The protective effects reflected on greater PSII photochemical activity $(\mathrm{Fv} / \mathrm{Fm})$ and protected chloroplasts membranes from AOS attacks, and hence higher chlorophyll concentrations under water deficits were noted. Impact of drought stress imposed during growth stages R1-R3 on soybean seed vigor was greater than the effect during other growth stage. However, $\mathrm{Zn}$ application mitigated the negative effect of drought on produced seeds' quality and raised the mean germination rate considerably. In general, germination rate of $\mathrm{Zn}$-rich seeds was lower than the others; nevertheless, such seed lots had higher germination percentage and produced vigorous seedlings. Thus, it is recommended the application of zinc under $\mathrm{Zn}$ - and water-deficient soil conditions, to produce vigorous seed lots.

\section{References}

Ashraf M,ZafarZU, CheemaZA (1994).Effect of low potassium regimes on some salt and drought tolerant lines of pearl millet. Phyton 34:219227.

Aspinall D, Paleg LG (1981). Proline accumulation: Physiological aspects. In: Paleg LG, Aspinall D (Eds). Physiology and biochemistry of drought resistance in plants. Academic Press, New York pp 205-240.

Bai BZ (1992). Plant Physiology Beijing: China Agricultural Press.

Bonilla I, El-Hamdaoui A, Bolanos L (2004). Boron and calcium increase pea (Pisum sativum L.) seed germination and seedling development under salt stress. Plant and Soil 267(1-2):97-107.

Bort J, Araus JL, Hazzam H, Grando S, Ceccarelli S (1998). Relationships between early vigor, grain yield, leaf structure and stable isotope composition in field grown barley. Plant Physiology and Biochemistry 36(12):889-897.

Boutraa T, Senders FE (2001). Influence of water stress on grain yield and vegetative growth of two cultivars of bean (Phaseolusvulgaris $\mathrm{L}$ ). Journal of Agronomy and Crop Science 187(4):251-257.

Bowler C, Van Montagu M, Inze D (1992). Superoxide dismutase and stress tolerance. Annual Review of Plant Biology 43(1):83-116. 
190

Bradford M (1976). A rapid and sensitive method for the quantitation of microgram quantities of protein utilizing the principle of protein-dye binding. Analytical Biochemistry 72(1-2):248-254.

Bukhov NG (2004). Dynamic light regulation of photosynthesis. (A aeview). Russian Journal of Plant Physiology 51(6):742-753.

Chaves MM, Maroco JP, Pereira JS (2003). Understanding plant responses to drought - from genes to the whole plant. Functional Plant Biology 30(3):239-264.

Cakmak I, Marschner H, Bangerth F (1989). Effect of zinc nutritional status on growth, protein metabolism and levels of indole-3-acetic acid and other phytohormones in bean (Phaseolus vulgaris L.). Journal of Experimental Botany 40(3):405-412.

Cakmak I (2000). Possible roles of zinc in protecting plant cells from damage by reactive oxygen species. New Phytologist 146(2):185-205.

Cakmak I (1994). Activity of ascorbate-dependent H2O2-scavenging enzymes and leaf chlorosis are enhanced in magnesium and potassium deficient leaves but not in phosphorus deficient leaves. Journal of Experimental Botany 45(9):1259-1266.

Cakmak I (2008). Enrichment of cereals with zinc: agronomic or genetic biofortification? Plant and Soil 302(1-2):1-17.

Cakmak I, Engels C (1999). Role of mineral nutrients in photosynthesis and yield formation. Mineral nutrition of crops: Mechanisms and implications. The Haworth Press, New York, USA pp 141-168.

Cakmak I, Marschner H (1988). Increase in membrane permeability and exudation in roots of zinc deficient plants. Journal of Plant Physiology 132(3):356-361.

Cakmak I, Marschner H (1993). Effect of zinc nutritional status on activity of superoxide radical and hydrogen peroxide scavenging enzymes in bean leaves. Plant and Soil 155-156(1):127-130.

Castaneda SMC, Cordova TL, Gonzalez HTV, Delgoda AA, Santacruz VA ,Garcia de los SG (2006). Respuestas fisiologicas, rendimiento y calidad de semilla en frijol sometido a stress hidrico. Interciencia 31:461466.

Cornic G, Briantais JM (1991). Partitioning of photosynthetic electron flow between $\mathrm{CO}_{2}$ and $\mathrm{O}_{2}$ reduction in a $\mathrm{C}_{3}$ leaf (Phaseolus vulgaris $\mathrm{L}$.) at different $\mathrm{CO}_{2}$ concentrations and during drought stress. Planta 183(2):178-184.

Crowe JH, Crowe LM, Carpenter JF, Rudolph AS, Wistrom CA, Spargo BJ, Anchordoguy TJ (1988). Interactions of sugars with membranes. Biochimica et Biophysica Acta 947(2):367-384.

Cruz-Aguado J, Rodes R, Perez I, Dorado M (2000). Morphological characteristics and yield components associated with accumulation and loss of dry mass in the internodes of wheat. Field Crop Research 66(2):129-139.

Erenoglu B, Nikolic M, Römhold V, Cakmak I (2002). Uptake and transport of foliar applied zinc $(65 \mathrm{Zn})$ in bread and durum wheat cultivars differing in zinc efficiency. Plant and Soil 241(2):251-257.

Farahat MMM, Soad Ibrahim MM, Taha LS, FatmaEl-Quesni EM (2007). Response of vegetative growth and some chemical constituents of Cupressus sempervirens $\mathrm{L}$. to foliar application of ascorbic acid and zinc at Nubaria. WorldJournal of Agricultural Sciences 3(4):496-502.

Gadallah MAA (2000). Effects of indole-3- acetic acid and zinc on growth, osmotic potential and soluble carbon and nitrogen components of soybean plants growing under water deficit. Journal of Arid Environments $44(4): 451-467$.
Garg BK, Vyas SP, Kathju S, Lahiri AN (1998). Influence of water deficit at various growth stages on some enzymes of nitrogen metabolism and yield in cluster bean genotypes. Indian Journal of Plant Physiology 3(3):214 218.

Giannopolitis C, Ries S (1997). Superoxid dismutase I. Occurence in higher plant. Plant Physiology 59:309-314.

Graham RD, Rengel Z (1993). Genotypic variation in zinc uptake and utilization by plants. In: Robson $\mathrm{AD}(\mathrm{Ed})$.Zinc in soils and plants. Kluwer Academic Publishers, Dordrecht, Springer Netherlands pp 107-118.

Graham RD, Senadhira D, Beebe SE, Iglesis C (1997). A strategy for breeding staple-food crops with high micronutrient density. Plant nutrition for sustainable food production and environment. Springer Netherlands 933937.

Grossman A, Takahashi H (2001). Micronutrient utilization by photosynthetic eukaryotes and the fabric of interactions. Annual Review of PlantBiology 52(1):163-210.

Hatch MD, Slack CR(1970). Photosynthetic $\mathrm{CO}_{2}$ fixation pathways. Annual Review of Plant Physiology 21(1):141-162.

Hsiao TC (1973). Plant responses to water stress. Annual Review of Plant Physiology24(1):519-570.

$\mathrm{Hu}$ Y, Schmidhalter U (1998). Spatial distributions of inorganic ions and carbohydrates contributing to osmotic adjustment in the elongating wheat leafunder saline soil conditions. Functional Plant Biology 25(5):591-597.

Kitagishi K, Obata H (1986). Effects of zinc deficiency on the nitrogen metabolism of meristematic tissues of rice plants with reference to protein synthesis.Soil Science and Plant Nutrition 32(3):397-405.

Khan HR, McDonaldGK, RengelZ (2003).Zinc fertilization improves water use efficiency, grain yield and seed $\mathrm{Zn}$ content in chickpea. Plant and Soil 249(2):389-400.

Khan HR, McDonaldGK, RengelZ(2004).Zinc fertilization and water stress affects water relations, stomatal conductance and osmotic adjustment in chickpea (Cicer arietinum L.).Plant andSoil 267(1-2):271-284.

Kokubun M, Shimada S, Takahashi M (2001). Flower abortion caused by preanthesis water deficit is not attributed to impairment of pollen soybean. CropScience 41(5):1517-1521.

LuC,ZhangJ (1999). Effects of water stress on photosystem II photochemistry and its thermostability in wheat plants. Journal of Experimental Botany 50(336):1199-1206.

Luna M, Badiani M, Felice M, Artemi F, Germanni G (1985). Selective enzyme inactivation under water stress in maize (Zea mays $\mathrm{L}$.) and wheat (Triticum aestium L.) seedlings. Enviromental and Experimental Botany 25(2):153-156.

Fuhrer J (2003). Agroecosystem responses to combinations of elevated CO2, ozone, and global climate change. Agriculture, Ecosystems and Environment 97(1):1-20.

Fischer ES (1997). Moderate magnesium deficiency affected chlorophyll content of bean plat. Photosynthetica 33:385-390.

Marschner H (1993). Mineral nutrition of higher plants. Second edition. New York. Academic Press, USA.

Marschner H (1995). Mineral nutrition of higher plants. Second edition. Academic Press, San Diego.

Michauil T, Walter T, Astrid W, Walter G, Dieter G, Maria SJ, Domingo M (2004). A survey of foliar mineral nutrient concentrations of Pinus canariensis at field plots in Tenerife. Forest Ecology and Management 189(1):49-55. 
Movahhedy-Dehnavy M, Modarres Sanavy SAM, Mokhtassi-Bidgoli A (2009). Foliar application of zinc and manganese improves seed yield and quality of safflower (Carthamus tinctorius L.) grown under water deficit stress. Industrial Crops and Products 30(1):82-92.

Mullet JE, Whitsitt MS (1996). Plant cellular responses to water deficit. Drought tolerance in higher plants: Genetical, physiological and molecular analysis. SpringerNetherlands pp 4146.

Pedroza FJA, Munoz OA (1993). Resistencia ontogenica y filogenetica a sequira en Phaseolus vulgaris L. I. Caracteres vegetativos. Agrociencia Ser Fiticiencia 4:19-33.

Perry DA (1980). The concept of seed vigor and its relevance to seed production techniques. In: Hebblethwaite PD (Ed). Seed Production, Butterworths, London pp 584591.

Ragoi $\mathrm{V}$ (1994). Changes in free amino acids and osmotic adjustment in leaves of water stressed bean. Physiologia Plantarum 91(3):427434.

Rassini JB, Lin SS (1981). Efeitos de periodos de estiagens artificais durante estadios de desenvolvimento da planta no rendimento e qualidade da semente de soja (Glycine $\max (\mathrm{L}$ ) Merr.). Agronomy Sulriograndense1 17:225-237.

Razmjoo K, Henderlong PR (1997). Effect of potassium,sulfur, boron, and molybdenum fertilization on alfalfa production and herbage macronutrient contents.Journal of Plant Nutrition 20(12):1681-1696.

Savitskaya NN (1976). On the physiological role of the proline in plants. Biol Nauki(Moscow) 19:49-61.

Singh K, Ghosal G, Singh J (1992). Effect of sulphur, zinc and iron on chlorophyll content, yield, protein harvest and nutrient uptake of French bean (Phaseolus vulgaris L.). Journal of Plant Nutrition 15(10):2025-2033.

Sharma PN, Tripathi A, Bisht SS (1995). Zinc requirement for stomatal openingin cauliflower. Plant Physiology 107(3):751-756.

Shen XY, Webster DB (1986). Effect of water stress on pollen of Phaseolus vulgaris L. Journal of the American Society for Horticultural Science 111(5):807-810.

Smirnoff N (1995). Antioxidant systems and plant response to the envirionment. In: Smirnoff V (Ed). Environment and plant metabolism: flexibility and acclimation. Oxford Bios Scientific Publisherspp 217-243.

Taiz L, Zeiger E (1991). Plant physiology. Benjumin- Cummingog, Redwood City, CA.

Thalooth AT, Tawfik MM, Magda Mahmed H (2006). A comparative study on the effect of foliar application of zinc, potassium and magnesium on growth, yield and some chemical constituents of mungbean plants grown under water stress conditions. World Journal of Agricultural Science 2(1):37-46.

Tavallali V, Rahemi M, Eshghi S, Kholdebarin B, Ramezanian A (2010). Zinc alleviates salt stress and increase antioxidant enzyme activity in the leaves of pistachio (Pistacia vera L. Badami) seedlings. Turkish Journal of Agriculture and Forestry34(4):349-359.

Thomas M J, Robertson SF, Peoples MB (2004). The effect of timing and severity of water deficit on growth, development, yield accumulation and nitrogen fixation of mungbean. Field CropsResearch 86(1):67-80.
Turkan I, Bor M, Ozdemir F, Koja H (2005). Differential responses of lipid peroxidation and antioxidants in the leaves of drought-tolerant $P$. acutifolius Gray and drought-sensitive $P$. vulgaris $\mathrm{L}$ subjected to polyethylene glycol mediated water stress. PlantScience 168(1):223-231.

Verkleij JAC, Schat H (1990). Mechanisms of metal tolerance in higher plants. In: Shaw $\mathrm{AJ}(\mathrm{Ed})$. Heavy metal tolerance in plantsevolutionary aspects. CRC Press, Boca Raton, FL pp 179-193.

Vieira RD, Tekrony DM, Egli DB (1992). Effect of drought and defoliation stress in the field in the on soybean seed germination and vigour. Journal of Crop Science 32(2):471-475.

Wang H, Liu RL, Jin JY (2009). Effects of zinc and soil moisture on photosynthetic rate and chlorophyll fluorescence parameters of maize. Biologia Plantarum 53(1):191-194.

Wang ZL, Huang BR (2004). Physiological recovery of Kentucky bluegrass from simultaneous drought and heat stress. Crop Science 44(5):1729-1736.

Wang H, Jin JY (2007). Effects of zinc deficiency and drought on plant growth and metabolism of reactive oxygen species in maize (Zea mays L.). Agricultural Science in China 6(8):988-995.

Westgate ME, Schussler JR, Reicosky DC, Brenner ML (1989). Effect of water deficits on seed development in soybean: II. Conservation of seed growth rate. Plant Physiology. 91(3):980-985.

White JG, Zasoski RJ (1999). Mapping soil micronutrients. Field Crops Research 60(1):11-26.

Yadav VK, Gupta V, Nyphlam Y (1999). Hormonal regulation of nitrate in gram (Cicer arietinum L.) genotypes under drought. The Indian Journal of Agricultural Sciences 69(8):592-595.

Yilmaz A, Ekiz H, Gültekin I, Torun B, Barut H, Karanlik S, Cakmak I (1998). Effect on seed zinc content on grain yield and zinc concentration of wheat grown in zinc-deficient calcareous soils. Journal of Plant Nutrition 21(10):2257-2264.

Yordanov I, Velikova V, Tsonev T (2003). Plant response to drought and stress tolerance. Bulgarian Journal of Plant Physilogy Special Issue:187-206.

Zayed BA, Salem AKM, El Sharkawy HM (2011). Effect of different micronutrient treatments on rice (Oriza sativa $L$.) growth and yield under saline soil conditions. World Journal of Agricultural Science 7(2):179-184.

Zinselmeier C, Lauer MJ, Boyer JS (1995). Reversing droughtinduced losses in grain yields. Sucrose maintains embryo growth in maize. Crop Science 35(5):1390-1400.

Zhang J, Kirkham MB (1996). Antioxidant responses to drought in sunflower and sorghum seedlings. New Phytologist 132(3):361-370.

Zhang J, Kirkham MB (1994). Drought-stress-induced changes in activities of superoxide dismutase, catalase, and peroxidase in wheat species. Plant and Cell Physiology 35(5):785-791. 